\title{
Nestin regulates epithelial-mesenchymal transition marker expression in pancreatic ductal adenocarcinoma cell lines
}

\author{
MASAHITO HAGIO, YOKO MATSUDA, TAEKO SUZUKI and TOSHIYUKI ISHIWATA \\ Departments of Pathology and Integrative Oncological Pathology, Nippon Medical School, Tokyo 113-8602, Japan
}

Received May 31, 2012; Accepted June 4, 2012

DOI: $10.3892 / \mathrm{mco} .2012 .1$

\begin{abstract}
Nestin, a class VI intermediate filament, is a neuronal stem/progenitor cell marker that is also expressed by various types of cancer, including pancreatic ductal adenocarcinoma (PDAC). We previously detected nestin expression in approximately $30 \%$ of PDAC cases, and found that nestin promotes the migration, invasion and metastasis of cells. Findings of recent studies have shown that epithelial mesenchymal transition (EMT) is important in the invasion and migration of cancer. In the present study, we investigated whether an altered nestin expression affected the expression levels of EMT markers in PDAC cells. Two human PDAC cell lines, PK-45H and KLM-1, in which nestin was suppressed and overexpressed, respectively, were used. The expression levels of the EMT-related molecules E-cadherin, Snail, Slug and Twist were analyzed using quantitative RT-PCR. Results showed that E-cadherin expression was decreased in nestin-overexpressed KLM-1 cells, and increased in nestin-suppressed PK-45H cells. Snail gene expression in the PDAC cells was altered concomitantly with the changes in nestin expression, while the Slug gene expression was significantly decreased in nestin-overexpressed KLM-1 cells. The Twist gene expression was below the detection limit in the two PDAC cell lines. The present findings indicated that nestin may be involved in the control of cancer behaviors in PDAC via the modulation of EMT-related molecules.
\end{abstract}

\section{Introduction}

Pancreatic ductal adenocarcinoma (PDAC) is associated with extremely high mortality rates due to rapid progression and high incidences of metastases, peritoneal dissemination and recurrence (1). Therefore, there is an urgent need for new therapeutic strategies that are focused on preventing the invasion and metastasis of pancreatic cancer cells.

Correspondence to: Dr Toshiyuki Ishiwata, Departments of Pathology and Integrative Oncological Pathology, Nippon Medical School, 1-1-5 Sendagi, Bunkyo-ku, Tokyo 113-8602, Japan

E-mail: ishiwata@nms.ac.jp

Key words: nestin, epithelial mesenchymal transition, pancreatic cancer, Snail, Slug
Nestin, a class VI intermediate filament, was first described as a neuronal stem/progenitor cell marker that is expressed in progenitor cells of various tissues, including pancreas (2). Lineage-tracing experiments have shown that exocrine cells in the pancreas are derived from nestin-expressing progenitor cells (3-5). An increased nestin expression has been reported in various tumors, including pancreatic cancer (6). Nestin immunoreactivity was found to be present in the cancer cells in approximately $30 \%$ of PDAC cases. Moreover, nestin expression in pancreatic cancer cells was found to correlate with nerve invasion and the presence of cancer cells at the tumor resection margins (7). Suppression of the nestin expression was found to inhibit the invasion and migration of PDAC cells in vitro, and inhibit liver metastasis in a xenograft mouse model (8). These findings suggest that nestin is important in the aggressiveness of pancreatic cancer cells.

The epithelial-mesenchymal transition (EMT), in which cells undergo a morphological switch from the epithelial polarized phenotype to the mesenchymal fibroblastoid phenotype, is considered to occur during cancer invasion and metastasis (9). Several distinct traits are conveyed by EMT, including cell motility and invasiveness (10). As a result of EMT, epithelial cells lose their defined cell-cell/ cell-substratum contacts and their structural/functional polarity, and become spindle-shaped and morphologically similar to activated fibroblasts (11). At the molecular level, EMT is defined by the loss of cell-cell adhesion molecules (e.g., E-cadherin and ZO-1), downregulation of epithelial differentiation markers (e.g., cytokeratins and E-cadherin), transcriptional induction of mesenchymal markers (e.g., vimentin, fibronectin, and $\mathrm{N}$-cadherin) and the nuclear localization of $\beta$-catenin (12). E-cadherin plays a major role in EMT, and the Snail, Twist, and SIP-1/ZEB-2 proteins are all repressors of the gene $\mathrm{CDH} 1$ that codes for E-cadherin.

We hypothesized that nestin regulates the migration and invasion of PDAC cells via interactions with EMT factors. In the present study, we modulated the nestin expression in PDAC cells and investigated the corresponding changes in the mRNA and protein levels of major EMT factors.

\section{Materials and methods}

Reagents and chemicals. The following reagents and chemicals were purchased: FuGene HD transfection reagent from Roche Diagnostics (Mannheim, Germany); geneticin from Gibco-BRL 
(Grand Island, NY, USA); pBAsi-hU6 Neo DNA vector and FastPure RNA kit from Takara Bio, Inc. (Tokyo, Japan); pAcGFP1-N1 vector from Clontech Laboratories (Mountain View, CA, USA) and the High Capacity cDNA Reverse Transcription kit. TaqMan Fast Universal PCR Master Mix, and TaqMan Gene Expression Assays for nestin (Hs00707120_s1), E-cadherin (Hs01013953_m1), Snail (Hs00195591_m1), Slug (Hs00950344_m1), Twist (Hs00361186_m1), and 18S rRNA (Hs99999901_s1) were obtained from Applied Biosystems, Inc. (Foster City, CA, USA). Rabbit polyclonal anti-Snail antibody was purchased from Abcam (Cambridge, UK); Alexa 488-labeled goat anti-rabbit IgG antibody was purchased from Invitrogen Life Technologies (Carlsbad, CA, USA); and Vectashield H-1200 containing 4',6-diamidino-2-phenylindole-2HCl (DAPI) was obtained from Vector Laboratories (Burlingame, CA, USA). All other chemicals and reagents were purchased from Sigma-Aldrich (St. Louis, MO, USA).

Pancreatic cancer cell lines. PK-45H and KLM-1 human pancreatic cancer cells were obtained from the Cell Resource Center for Biomedical Research, Institute of Development, Aging, and Cancer, Tohoku University, Japan. Cells were grown in RPMI-1640 medium containing $10 \%$ fetal bovine serum (FBS) at $37^{\circ} \mathrm{C}$ under a humidified $5 \% \mathrm{CO}_{2}$ atmosphere.

Establishment of nestin short hairpin RNA-transfected PK-45H cells. Human nestin short hairpin (sh) RNA was prepared as previously reported (8). PK-45H cells $\left(1 \times 10^{5}\right.$ cells/well) were plated in 6-well plates and grown in $2 \mathrm{ml}$ RPMI-1640 medium with $10 \%$ FBS. Transfections of the nestin shRNA expression and sham vectors were performed using FuGENE HD transfection reagent, according to the manufacturer's instructions. Independent colonies were isolated by ring cloning, and expanded in $300 \mu \mathrm{g} / \mathrm{ml}$ geneticin. Cell lysates were collected, and nestin mRNA was measured by quantitative RT-PCR (qRT-PCR).

Establishment of nestin-expressed vector-transfected KLM-1 cells. The full-length nestin cDNA fragment was ligated into the pAcGFP1-N1 eukaryotic expression vector as reported in a previous study (8). Transfections with the nestin expression vector (Nes) and the empty vector (Mock) were performed using FuGENE HD transfection reagent. The cells were passaged and cultured with $600 \mu \mathrm{g} / \mathrm{ml}$ geneticin. Independent colonies were isolated by ring cloning and expanded in $300 \mu \mathrm{g} / \mathrm{ml}$ geneticin.

$q R T-P C R$. Total RNA was extracted from cells and purified with the FastPure RNA kit. One microgram of the total RNA sample was used for reverse transcription (RT) with the High Capacity cDNA Reverse Transcription kit following the manufacturer's protocol. qRT-PCR for nestin, E-cadherin, Snail, Slug, Twist and 18S rRNA was performed with the StepOnePlus RealTime PCR System (Applied Biosystems, Inc.) using specific primers and a TaqMan probe. Cycling conditions were as follows: denaturation for $20 \mathrm{sec}$ at $95^{\circ} \mathrm{C}$, followed by annealing for 40 cycles of $1 \mathrm{sec}$ at $95^{\circ} \mathrm{C}$ and a final extension for $20 \mathrm{sec}$ at $60^{\circ} \mathrm{C}$. qRT-PCR results were expressed as the ratio of the target to $18 \mathrm{~S}$ rRNA, the latter serving as an internal standard. Gene expression levels were measured in triplicate.
Immunocytochemistry. Cells were plated in $35-\mathrm{mm}$ glassbottomed dishes $\left(2 \times 10^{5}\right.$ cells/dish) and incubated for $72 \mathrm{~h}$ at $37^{\circ} \mathrm{C}$ in a humidified $5 \% \mathrm{CO}_{2}$ atmosphere. The cells were fixed in $4 \%$ paraformaldehyde solution for $15 \mathrm{~min}$ at room temperature, and incubated overnight at $4{ }^{\circ} \mathrm{C}$ with polyclonal rabbit anti-Snail antibody. Cells were then incubated with Alexa 488-labeled anti-rabbit IgG antibody (1:1000 dilution), and mounted in Vectashield H-1200. Snail protein was visualized using a Digital Eclipse C1 TE2000-E microscope (Nikon Instech Co., Ltd., Tokyo, Japan). Fluorescent images were analyzed using control software EZ-C1 (Nikon). Confocal settings, including the laser power and detector sensitivity, were unchanged during the acquisition of all images.

Statistical analysis. Quantitative data were shown as the mean \pm SEM. One-way ANOVA was used to compare data for each shRNA clone separately for the corresponding values for each of two sham clones. $\mathrm{P}<0.05$ was considered to indicate a statistically significant difference with respect to each of the two sham clones. Data for transiently nestin gene-transfected cells and corresponding Mock-transfected cells were assessed by the Student's t-test. Computations were performed using the Stat View J version 5.0 software package (SAS Institute, Inc., Cary, NC, USA).

\section{Results}

Increase and decrease in expression levels of nestin and E-cadherin in PK-45H and KLM-1 cells. Nestin mRNA expression levels in PK-45H and KLM-1 cells were analyzed using qRT-PCR (Fig. 1). In PK-45H cells, nestin shRNA-transfected clones (Sh-7, -8, and -9) exhibited a markedly decreased nestin expression compared with the sham vector-transfected cells (Sc-13 and -17) (Fig. 1A). Sh-7 cells showed the lowest nestin expression, approximately $40 \%$ lower than that of Sc-13 cells. By contrast, KLM-1 cells originally expressed low levels of nestin, and nestin-expressed vector-transfected KLM-1 cells (Nes-9 and -12) expressed higher nestin levels than the empty vector-transfected clones (Mock-13 and -24) (Fig. 1B). Nes-12 cells showed the highest nestin expression level, which was approximately eight times the level of Mock-24 cells.

Furthermore, we examined the expression of EMT markers, including E-cadherin, a major marker for epithelial cells (Fig. 1). The nestin-suppressed PK-45H clones exhibited a markedly increased E-cadherin expression (Fig. 1C). By contrast, the nestin-overexpressed KLM-1 clones showed significantly decreased E-cadherin mRNA levels (Fig. 1D).

$m R N A$ expression and immunostaining of Snail in PK-45H and KLM-1 cells. To investigate whether altered nestin expression levels affected E-cadherin expression through transcriptional factors, we analyzed the expression of Snail, Slug and Twist. However, Twist expression was not high enough to detect, which is consistent with previous findings (13). Suppression of nestin expression significantly decreased the Snail mRNA expression in PK-45H cells compared with that in sham-transfected Sc clones (Fig. 2A). In addition, the Snail protein expression was apparently decreased in the cytoplasm and nucleus of Sh-9 cells (Fig. 2C). By contrast, Snail mRNA expression was significantly higher in the nestin- 
A

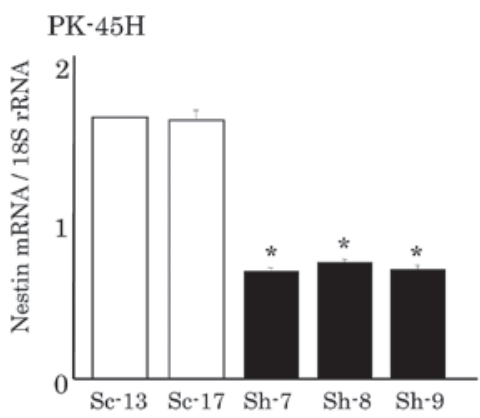

C

PK-45H

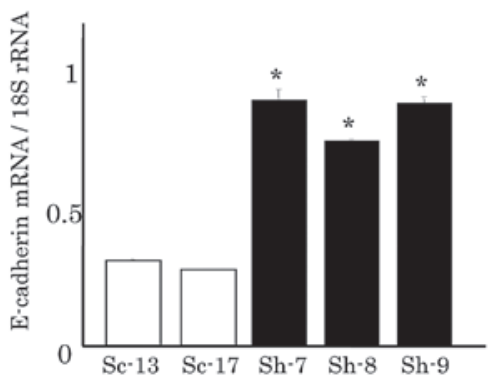

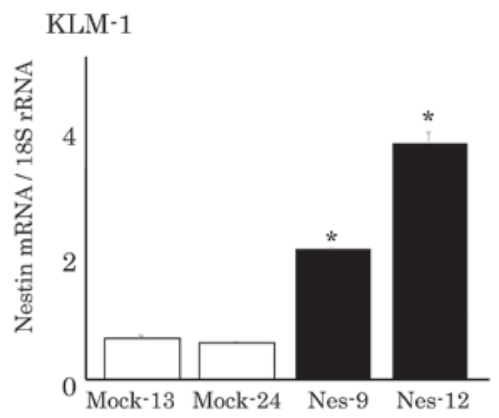

D

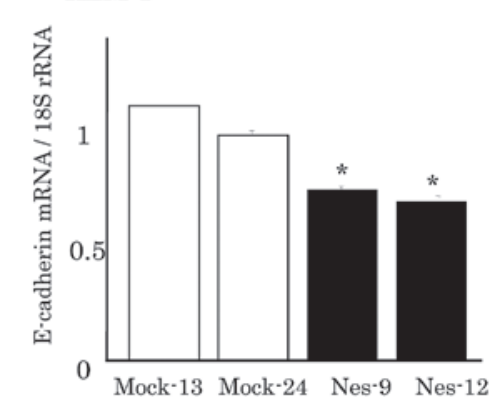

Figure 1. Expression of nestin and E-cadherin mRNAs in PK-45H and KLM-1 cells. (A) Sh clones exhibited a significantly decreased nestin mRNA expression compared with the Sc clones. (B) Nes clones exhibited a significantly increased nestin mRNA expression compared with the Mock clones. (C) Sh clones exhibited a significantly increased E-cadherin mRNA expression compared with the Sc clones. (D) Nes clones exhibited a significantly decreased E-cadherin mRNA expression compared with the Mock clones. ${ }^{*} \mathrm{P}<0.05$.

A

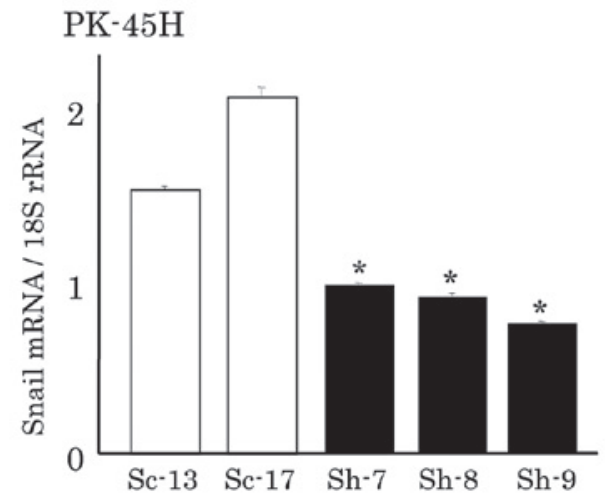

C

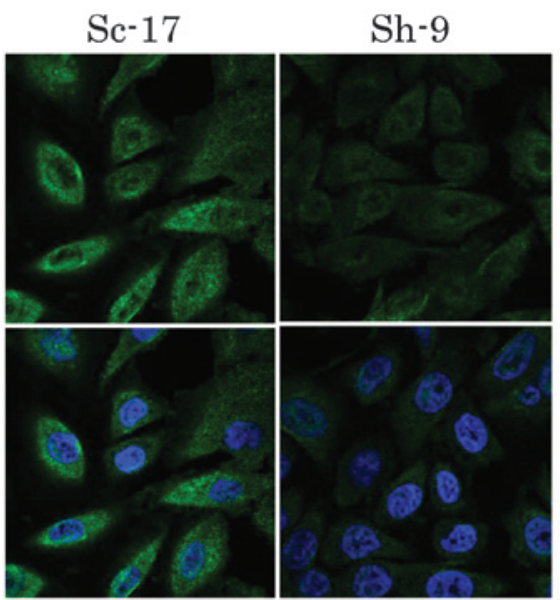

B

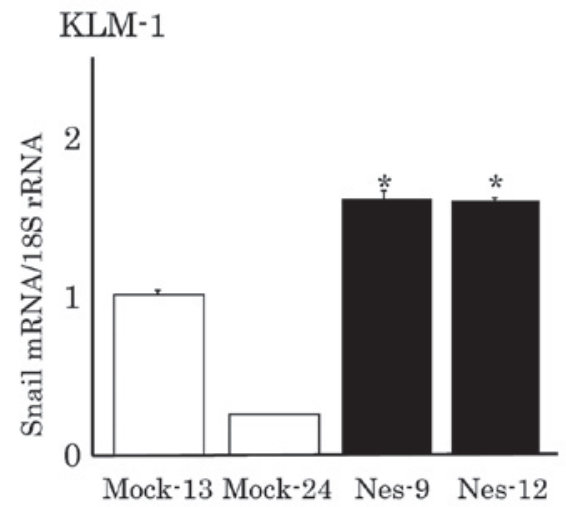

D

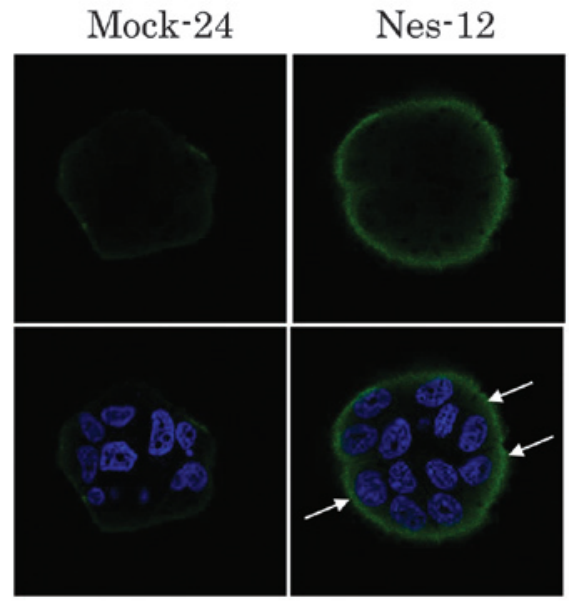

Figure 2. Snail expression in PK-45H and KLM-1 cells. (A and C) Sh clones exhibited a significantly decreased (A) Snail mRNA and (C) protein expression compared with the Sc clones. (B and D) Nes clones exhibited a significantly increased (B) Snail mRNA and (D) protein expression compared with teh Mock clones. "P<0.05. (A and B) qRT-PCR. (C and D) Immunocytochemistry: green, Snail; blue, DAPI. Upper panels, image of Snail; lower panels, merged image. Original magnification, $\mathrm{x} 1000$. 
A

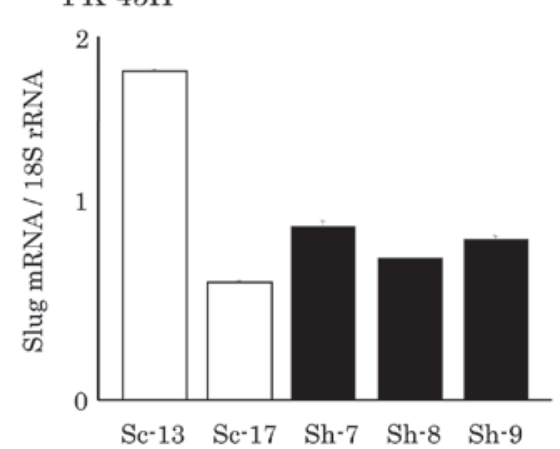

B

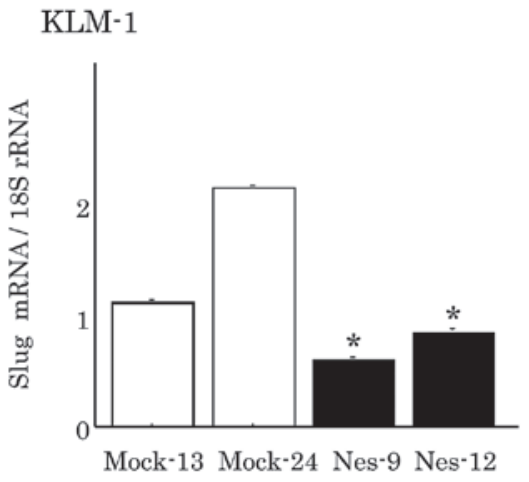

Figure 3. Expression of Slug mRNA in PK-45H and KLM-1 cells. (A) Slug expression was not statistically different between the Sc and Sh clones. (B) Nes clones exhibited a significantly decreased Slug mRNA expression compared with the Mock clones. ${ }^{*} \mathrm{P}<0.05$.

expressed vector-transfected KLM-1 cells compared to the Mock-transfected cells (Fig. 2B). Similarly, an increased Snail protein expression was detected in these cells (Nes-9 and -12) (Fig. 2D).

mRNA expression of Slug in PK-45H and KLM-1 cells. Slug expression was not significantly different between the Sc and nestin-suppressed Sh PK-45H clones (Fig. 3A). However, the nestin-overexpressed KLM-1 cells showed a significantly decreased Slug mRNA expression (Fig. 3B).

\section{Discussion}

The correlation between the cytoskeletal proteins nestin and E-cadherin in cancer cells remains to be elucidated. Thyroid carcinomas exhibit a high nestin expression, while E-cadherin expression is not detected in anaplastic (undifferentiated) carcinoma. By contrast, E-cadherin, but not nestin, is expressed in papillary and follicular carcinomas (14). The association between these proteins in pancreatic cancer has yet to be adequately clarified. Results of our previous study have shown an increased E-cadherin expression in nestin-suppressed PDAC cells (8). Findings of the present study have shown that nestin overexpression induced the E-cadherin expression, strengthening the association between nestin and E-cadherin.

EMT occurs during various developmental processes, such as gastrulation, neural crest migration and heart formation (15-18). EMT is also involved in pathological processes, such as fibrosis and metastasis (19). The molecular mechanisms controlling EMT have only recently begun to emerge, and key roles have been identified for the zinc finger transcription factors Snail (Snail1) and Slug (Snail2) (20,21). In the present study, E-cadherin was negatively correlated with Snail expression, which is consistent with previous reports that indicate Snail is a repressor of E-cadherin and directly regulates E-cadherin expression via the integrin-linked kinase (ILK) pathway (22). We observed that the Snail expression responded to the increase and decrease of nestin, indicating that nestin is closely correlated with EMT and may thus modulate EMT.

Slug was found to behave differently to Snail. In chicks, Slug has been identified in two developmental processes involving EMT, as a key regulator of mesoderm formation and neural crest migration (23). Another study reported differential effects of Snail and Slug in pancreatic cancer, relating to the interplay between Rho signaling and $\beta 1$-integrin, and leading to differences in cell migration and scattering (24). Our findings suggest that Slug expression is less involved in EMT via the nestin-induced change of E-cadherin. Twist was not detected in this study; however, it may be involved. Twist reportedly may act through a different mechanism compared to Snail and Zeb proteins (25), and immunohistochemical analysis has detected a decreased expression of nuclear Twist in malignant pancreatic epithelium (26).

In conclusion, the results of this study suggest that nestin may modulate the expression of E-cadherin and Snail, thereby affecting the migration and metastasis of pancreatic cancer.

\section{Acknowledgements}

The authors thank Dr Z. Naito for his helpful discussion and Ms. K. Kawahara, Mr. Y. Yanagisawa and Ms. Y. Kawamoto (Department of Pathology, Integrative Oncological Pathology) for their excellent technical assistance. This study was supported by a Grant-in-Aid for Young Scientists (A, no. 22689038 to Y. Matsuda and B, no. 24791451 to M. Hagio), a Grantin-Aid for Challenging Exploratory Research (no. 23650604 to Y. Matsuda) and a Grant-in-Aid for Scientific Research (C, no. 22591531 to T. Ishiwata) from the Japan Society for the Promotion of Sciences. This study was also supported by a grant from the Pancreas Research Foundation of Japan to M. Hagio.

\section{References}

1. Jemal A, Siegel R, Ward E, Hao Y, Xu J, Murray T and Thun MJ: Cancer statistics, 2008. CA Cancer J Clin 58: 71-96, 2008.

2. Lendahl U, Zimmerman LB and McKay RD: CNS stem cells express a new class of intermediate filament protein. Cell 60: 585-595, 1990.

3. Esni F, Stoffers DA, Takeuchi T and Leach SD: Origin of exocrine pancreatic cells from nestin-positive precursors in developing mouse pancreas. Mech Dev 121: 15-25, 2004.

4. Delacour A, Nepote V, Trumpp A and Herrera PL: Nestin expression in pancreatic exocrine cell lineages. Mech Dev 121: 3-14, 2004.

5. Ishiwata T, Kudo M, Onda M, Fujii T, Teduka K, Suzuki T, Korc $\mathrm{M}$ and Naito Z: Defined localization of nestin-expressing cells in L-arginine-induced acute pancreatitis. Pancreas 32: 360-368, 2006 
6. Parry S, Savage K, Marchio C and Reis-Filho JS: Nestin is expressed in basal-like and triple negative breast cancers. J Clin Pathol 61: 1045-1050, 2008.

7. Carrière C, Seeley ES, Goetze T, Longnecker DS and Korc M: The Nestin progenitor lineage is the compartment of origin for pancreatic intraepithelial neoplasia. Proc Natl Acad Sci USA 104: 4437-4442, 2007.

8. Matsuda Y, Naito Z, Kawahara K, Nakazawa N, Korc M and Ishiwata T: Nestin is a novel target for suppressing pancreatic cancer cell migration, invasion and metastasis. Cancer Biol Ther 11: 512-523, 2011.

9. Micalizzi DS, Farabaugh SM and Ford HL: Epithelialmesenchymal transition in cancer: parallels between norma development and tumor progression. J Mammary Gland Biol Neoplasia 15: 117-134, 2010.

10. Larue L and Bellacosa A: Epithelial-mesenchymal transition in development and cancer: role of phosphatidylinositol 39 kinase/ AKT pathways. Oncogene 24: 7443-7454, 2005.

11. Hay ED: The mesenchymal cell, its role in the embryo, and the remarkable signaling mechanisms that create it. Dev Dyn 233: 706-720, 2005

12. Casas E, Kim J, Bendesky A, Ohno-Machado L, Wolfe CJ and Yang J: Snail2 is an essential mediator of Twist1-induced epithelial mesenchymal transition and metastasis. Cancer Res 71: 245-254, 2011.

13. Hotz B, Arndt M, Dullat S, Bhargava S, Buhr HJ and Hotz HG: Epithelial to mesenchymal transition: expression of the regulators snail, slug, and twist in pancreatic cancer. Clin Cancer Res 13: 4769-4776, 2007.

14. Liu J and Brown RE: Immunohistochemical detection of epithelialmesenchymal transition associated with stemness phenotype in anaplastic thyroid carcinoma. Int J Clin Exp Pathol 3: 755-762, 2010.

15. Hay ED: An overview of epithelio-mesenchymal transformation. Acta Anat (Basel) 154: 8-20, 1995.

16. Birchmeier C, Birchmeier W and Brand-Saberi B: Epithelialmesenchymal transitions in cancer progression. Acta Anat (Basel) 156: 217-226, 1996.
17. Thiery JP: Epithelial-mesenchymal transitions in tumour progression. Nat Rev Cancer 2: 442-454, 2002.

18. Kalluri R and Neilson EG: Epithelial-mesenchymal transition and its implications for fibrosis. J Clin Invest 112: 1776-1784, 2003.

19. Taylor MA, Parvani JG and Schiemann WP: The pathophysiology of epithelial-mesenchymal transition induced by transforming growth factor-beta in normal and malignant mammary epithelial cells. J Mammary Gland Biol Neoplasia 15: 169-190, 2010.

20. Savagner P: Leaving the neighborhood: molecular mechanisms involved during epithelial-mesenchymal transition. Bioessays 23 912-923, 2001.

21. Huber MA, Kraut N and Beug H: Molecular requirements for epithelial-mesenchymal transition during tumor progression. Curr Opin Cell Biol 17: 548-558, 2005.

22. Schaeffer DF, Assi K, Chan K, Buczkowski AK, Chung SW, Scudamore CH, Weiss A, Salh B and Owen DA: Tumor expression of integrin-linked kinase (ILK) correlates with the expression of the E-cadherin repressor snail: an immunohistochemical study in ductal pancreatic adenocarcinoma. Virchows Arch 456: 261-268, 2010.

23. Nieto MA, Sargent MG, Wilkinson DG and Cooke J: Control of cell behavior during vertebrate development by Slug, a zinc finger gene. Science 264: 835-839, 1994.

24. Shields MA, Krantz SB, Bentrem DJ, Dangi-Garimella S and Munshi HG: Interplay between $\beta 1$-integrin and Rho signaling regulates differential scattering and motility of pancreatic cancer cells by snail and Slug proteins. J Biol Chem 287: 6218-6229, 2012.

25. Montserrat N, Gallardo A, Escuin D, Catasus L, Prat J, GutiérrezAvignó FJ, Peiró G, Barnadas A and Lerma E: Repression of E-cadherin by SNAIL, ZEB1, and TWIST in invasive ductal carcinomas of the breast: a cooperative effort? Hum Pathol 42: 103-110, 2011.

26. Cates JM, Byrd RH, Fohn LE, Tatsas AD, Washington MK and Black CC: Epithelial-mesenchymal transition markers in pancreatic ductal adenocarcinoma. Pancreas 38: e1-e6, 2009. 